\title{
Techniques for correcting velocity and density fluctuations of ion beams in ion inducti on accelerators
}

\author{
K. M. Woo \\ Physics Department, The Chinese University of Hong Kong, Hong Kong, China \\ S. S. Yu \\ Physics Department, The Chinese University of Hong Kong, Hong Kong, China, \\ and Lawrence Berkeley National Laboratory, Berkeley, California 94720, USA

\section{J. J. Barnard} \\ Lawrence Livermore National Laboratory, Livermore, California 94551-0808, USA, \\ and Lawrence Berkeley National Laboratory, Berkeley, California 94720, USA
}

(Received 22 October 2011; revised manuscript received 6 January 2013; published 28 June 2013)

It is well known that the imperfection of pulse power sources that drive the linear induction accelerators can lead to time-varying fluctuation in the accelerating voltages, which in turn leads to longitudinal emittance growth. We show that this source of emittance growth is correctable, even in space-charge dominated beams with significant transients induced by space-charge waves. Two correction methods are proposed, and their efficacy in reducing longitudinal emittance is demonstrated with three-dimensional particle-in-cell simulations.

DOI: 10.1103/PhysRevSTAB.16.062804

PACS numbers: $29.27 .-\mathrm{a}$

\section{INTRODUCTION}

In an induction linac for heavy ion fusion (HIF) and high energy density physics applications, a long flattop voltage pulse is applied to a series of individual accelerator cells. Since these applications require axial compression of the ion beams by more than an order of magnitude before delivery to a target, growth of the longitudinal emittance from fluctuations in the accelerator cell voltages generated by imperfections in the pulse power driver outputs is a critical issue. Longitudinal emittance is a crucial beam parameter for HIF since it determines the final bunch length of an ion beam for ignition (Ref. [1]). Beams of short bunch length of 10 nanoseconds or less are required for ignition at target (Ref. [2]). Precise bunch length and pulse shape depend on details of target designs. For some advanced designs (e.g. fast ignitions), much shorter bunches are required. For many applications in high energy density physics, a short bunch length is also desired. The short final bunch length requirements imply that the longitudinal emittance growth must be carefully managed from the source to target.

The sources of longitudinal emittance growth include finite temperature and imperfect beam extraction from ion sources, head-to-tail nonlinearities, and other nonlinear space-charge effects, as well as voltage fluctuations in

Published by the American Physical Society under the terms of the Creative Commons Attribution 3.0 License. Further distribution of this work must maintain attribution to the author( $s)$ and the published article's title, journal citation, and DOI. linear induction accelerators. We show that longitudinal emittance increases significantly with the number of acceleration gaps, as a result of the large final velocity fluctuation when the beam is accelerated successively by time-varying voltage fluctuation gaps. Since multigap acceleration of ion beam is commonplace in HIF, techniques to reduce the growth of longitudinal emittance are therefore desired.

Longitudinal emittance $\epsilon_{z}$ is a quantity to measure the beam quality (Ref. [3]) and is directly related to the pulse length. Normalized longitudinal emittance $\epsilon_{z n}$ is defined by multiplication of a factor $\beta \gamma$. In the absence of nonlinear forces and no transverse-longitudinal coupling, $\epsilon_{z n}$ is invariant upon accelerations of beams:

$$
\begin{gathered}
\epsilon_{z} \equiv \frac{4}{\left\langle v_{z}\right\rangle} \sqrt{\left\langle\Delta z^{2}\right\rangle\left\langle\Delta v^{2}\right\rangle-\langle\Delta z \Delta v\rangle^{2}}, \\
\epsilon_{z n} \equiv \beta \gamma \epsilon_{z} .
\end{gathered}
$$

$\Delta z$ is defined as the particle position relative to the beam center and $\Delta v_{z}$ is defined as the $z$ component of the particle velocity relative to the average beam velocity. For example, Fig. 1 shows a $v_{z}-z$ phase space configuration for a high generalized perveance $\mathrm{Rb}$ beam accelerated by a gap with a "pulse shape" time-varying voltage fluctuation. For this space-charge dominated beam, around the beam head and tail a strong self-electric field is induced, driving the charged particles near the beam head to move faster than rear particles, resulting in a large velocity difference, and this phenomenon is called head-to-tail nonlinearity. A gentle velocity variation in the beam body was observed 


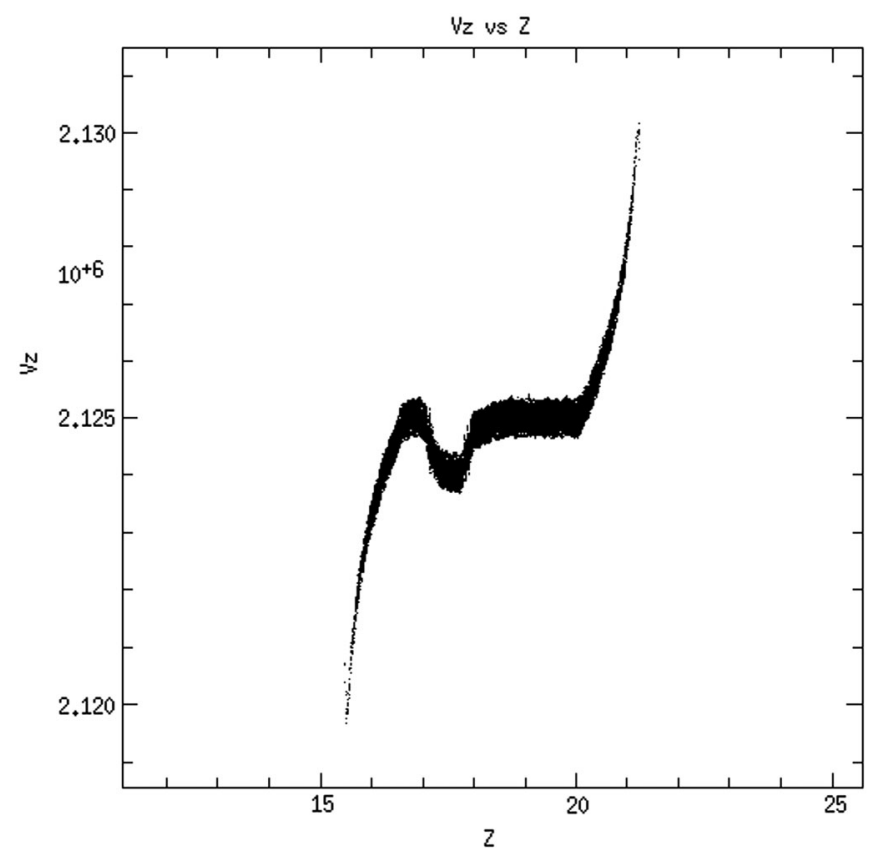

FIG. 1. An example to illustrate the disturbed phase space for a high generalized perveance $\mathrm{Rb}$ beam due to the head-to-tail nonlinearity and the gap voltage fluctuations.

due to the gap voltage fluctuation. These effects give rise to the big velocity spread $\Delta v_{z}$, resulting in large longitudinal emittance.

We concentrate on the parameter root-mean-square $\epsilon_{z n}$ rather than $\epsilon_{z}$, because in the absence of imperfections it remains constant not only during acceleration but also in the case of longitudinal ion beam compression such as the study of beams coasting with velocity tilt in Sec. II A.

Assume an ion beam with uniform line charge density over beam length $L$ and ion mass $m$ is extracted from the source, and the velocity spread is characterized by the source temperature $T$ according to the equipartition theorem. For axisymmetric beams, the term $\left\langle\Delta z \Delta v_{z}\right\rangle$ vanishes. For an ideal injector without any voltage fluctuation, the normalized longitudinal emittance $\epsilon_{z n, s}$ right after the ion beam extraction from the source is

$$
\epsilon_{z n, s}=\frac{4 L}{c \sqrt{12}} \sqrt{\frac{k_{B} T}{m}}
$$

Here $\sqrt{\left\langle\Delta z^{2}\right\rangle}=L / \sqrt{12}$ and $\sqrt{\left\langle\Delta v_{z}^{2}\right\rangle}=\sqrt{k_{B} T / m}$. In the presence of voltage fluctuations within the injector, the normalized longitudinal emittance $\epsilon_{z n, i}$ is (Ref. [1])

$$
\epsilon_{z n, i}=\frac{4}{m c} \tau E \delta .
$$

Here $\tau$ is the pulse length, $E$ the beam energy, and $\delta$ the fractional variation of voltage. In HIF a low source temperature $T$ favors the formation of final spot radius, so practically $\epsilon_{z n, s}$ is smaller than $\epsilon_{z n, i}$. On the basis of
Eq. (4), it has been argued that the pulse length at an HIF injector must be limited. This is of course untrue if this source of emittance growth can be corrected. The behavior of longitudinal emittance in multigap acceleration with time-varying voltage fluctuations is discussed in the paper and two techniques are proposed to reduce the longitudinal emittance growth by the intermittent application of correction voltages. Our objective is to remove both velocity and density fluctuations on the ion beams induced by the timevarying voltage fluctuations, arising from the imperfection of pulse power sources that drive the linear induction accelerators. The correction scheme is particularly nontrivial for space-charge dominated beams, on account of space-charge waves which propagate with time. We show by a combination of analyses and simulations with the $3 \mathrm{D}$ particle-in-cell code WARP that a significant reduction in longitudinal emittance was achieved.

\section{EFFECT OF GAP VOLTAGES ON $\epsilon_{z n}$}

Before we proceed to study the phenomenon of growing longitudinal emittance caused by voltage fluctuations, we first illustrate the conservation property of $\epsilon_{z n}$ by allowing a low current rubidium $(\mathrm{Rb})$ beam to coast inside a pipe under three different scenarios. This also provides a selfconsistency check for the code. All simulation scenarios in the entire paper involve three basic stages: (1) An unperturbed $\mathrm{Rb}$ beam is loaded, meaning that the initial $v_{z}-z$ phase space configuration of the input beam has the same kinetic energy over the pulse length with $0.1 \mathrm{eV}$ longitudinal thermal kinetic energy spread in Gaussian distribution, whereas the initial line charge density profile of the input beam has rounded ends with a flattop that extends $60 \%$ of the length of the beam, and with the flattop center coinciding with the beam center. (2) Then the beam is allowed to coast inside a long round pipe, made of periodic lattice cells, without hitting any boundary. (3) Finally the beam will be accelerated by gaps with 0.2 MV flattop voltage and different profiles of voltage fluctuations. The beam parameters in Table I are used in the entire paper.

TABLE I. Beam and lattice parameters. $\beta=7.08 \times 10^{-3}$; $\gamma \approx 1$; $*$ can be varied; see Refs. [4-6] for matched beam envelope calculations.

\begin{tabular}{lc}
\hline \hline Ion species & Rubidium $($ charge +1$)$ \\
\hline Injection energy & $2 \mathrm{MeV}$ \\
Injection current* & $0.02 \mathrm{~mA}$ \\
Initial unnormalized $\epsilon_{\perp}$ & $52 \times 10^{-6} \mathrm{~m} \mathrm{rad}$ \\
Generalized perveance* & $4.24 \times 10^{-8}$ \\
Mean beam radius & $1.07 \mathrm{~cm}$ \\
Nominal pulse length $\tau$ & $2.7 \mu \mathrm{s}$ \\
Period length & $72 \mathrm{~cm}$ \\
Phase advance & $18.65^{\circ}$ \\
Gap voltage $\Phi_{0}$ & $0.2 \mathrm{MV}$ \\
\hline \hline
\end{tabular}




\section{A. Effect of single constant voltage gap and a velocity-tilt beam}

When the ion beam is in transit through the gap, the beam is partially accelerated so that the velocity of the front part of the beam is much larger than the rear part, referred to the example of $v_{z}-z$ phase space configuration in Fig. 2(b). As a result, $\epsilon_{z n}$ is huge due to the presence of the big velocity variation according to Eqs. (1) and (2). However, this transient "bump" disappears when the beam completely leaves the gap. Since only the net growth of $\epsilon_{z n}$ due to effects of time-varying voltage fluctuation gaps is significant to our studies, the time evolution plots for the change of $\epsilon_{z n}$ before the beam enters the gap and after the beam completely leaves the gap are studied in the paper. Denote $t_{b}$ as the moment when the head of the beam is right at the entrance of the gap while $t_{a}$ denotes the moment when the tail of the beam is right at the exit of the gap. More explicitly, the measurement of the quantity $\Delta \epsilon_{z n}(t)$ is defined as

$$
\Delta \epsilon_{z n}(t)=\epsilon_{z n}\left(t \geq t_{a}\right)-\epsilon_{z n}\left(t_{b}\right) .
$$

Effects of nonlinearities and time-varying voltage fluctuations contribute to the positive growth of $\Delta \epsilon_{z n}$. Apart from Fig. 2(a), our studies of the time evolution of longitudinal emittance are based on the quantity $\Delta \epsilon_{z n}$. For convenience, we also studied the longitudinal emittance plots which only cover the period when the beam completely leaves the last gap, i.e. $\epsilon_{z n}\left(t \geq t_{a}\right)$.

The conservation property of $\epsilon_{z n}$ is illustrated in Fig. 2(a), which represents the scenario of passing a Rb beam through a gap with $0.2 \mathrm{MV}$ flattop voltage without any fluctuation. During the period approximately from 2 to $4.5 \mu \mathrm{s}$, a big bump is observed because the beam is in transit through the gap as explained previously. Apart from the bump, we found that $\epsilon_{z n}$ remains at constant value $3.2 \times 10^{-6} \pi \mathrm{m}$ rad before and after the gap, which illustrates that the normalized longitudinal emittance due to the thermal temperature at the source is conserved. This gives $\epsilon_{z n}\left(t_{b}\right)=\epsilon_{z n}(t=0)$ for the expression $\Delta \epsilon_{z n}(t)$, but this relation is not true for space-charge dominated beams due to the growing head-to-tail nonlinearity after the beam has left the source.

Figure 2(c) represents the scenario of coasting a $\mathrm{Rb}$ beam with a linear $10 \%$ velocity tilt inside a pipe without any acceleration gap. The velocity tilt is defined as

$$
\text { vtilt } \equiv \frac{v_{z}^{\text {tail }}-v_{z}^{\text {head }}}{v_{z}^{\text {beam }}} \times 100 \%
$$

Since the velocity tilt is positive, the beam is compressed so the pulse length decreases continually with time. In Fig. 2(c), the red crosses represent the compressed beam whereas the black curve represents the previous beam discussed in Fig. 2(a). The value of $\epsilon_{z n}(t=0)$ in both cases
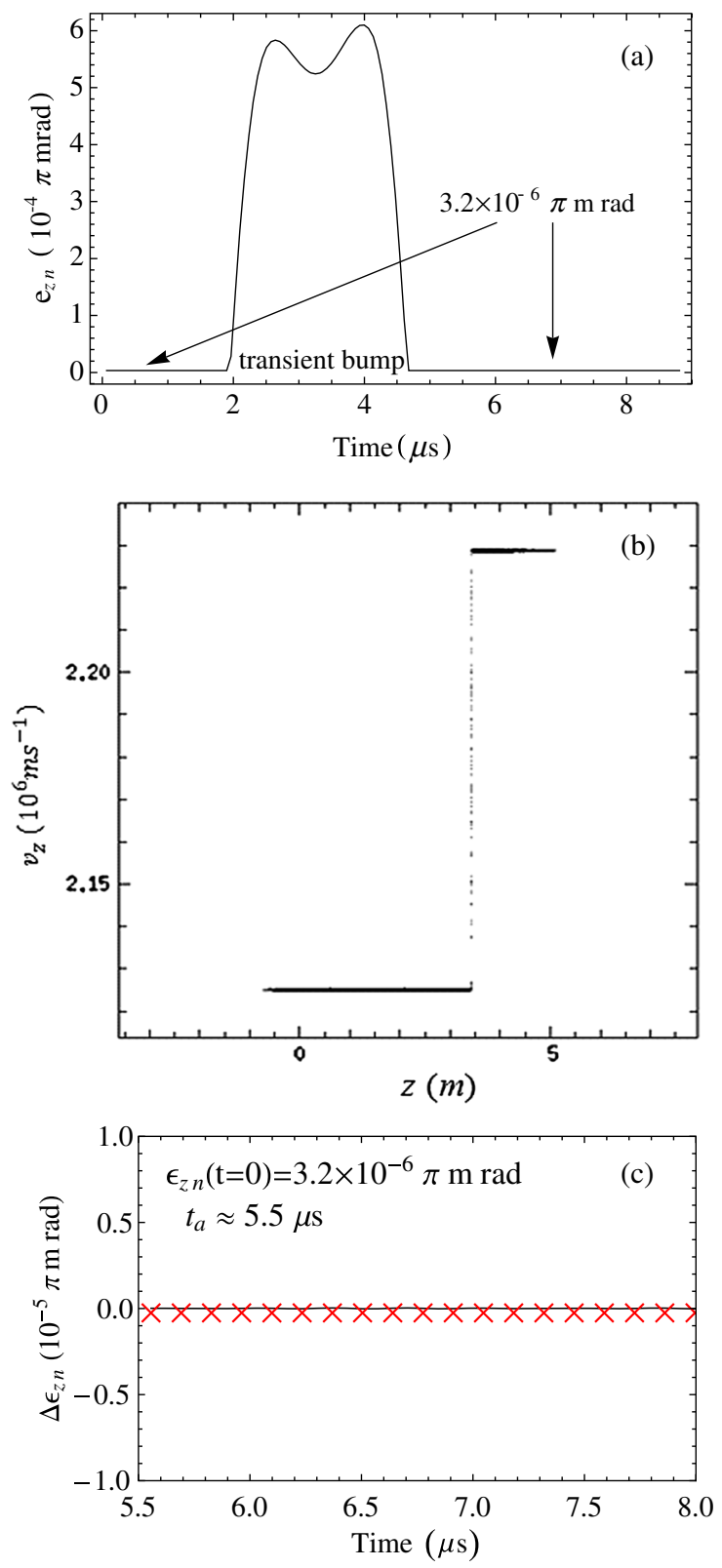

FIG. 2. (a) $\epsilon_{z n}(t)$ plot for the Rb beam accelerated by a gap with constant $0.2 \mathrm{MV}$ flattop voltage. (b) $v_{z}-z$ phase space configuration of the previous $\mathrm{Rb}$ beam in Fig. 2(a) to illustrate the big velocity difference when the beam is in transit through the gap. (c) The red crosses represent the $\Delta \epsilon_{z n}(t)$ plot for the compressed $\mathrm{Rb}$ beam with $10 \%$ velocity tilt. The black curve represents $\Delta \epsilon_{z n}(t)$ for previous beam in Fig. 2(a).

in Figs. 2(a) and 2(c) are the same as $3.2 \times 10^{-6} \pi \mathrm{m} \mathrm{rad}$ and the beam in Fig. 2(a) left the gap completely after time $t_{a}=3 \mu \mathrm{s}$. We found that $\Delta \epsilon_{z n}(t)$ remains nearly zero in both cases, meaning that the normalized longitudinal emittance of a compressed beam conserves. In general rootmean-square $\epsilon_{z n}$ conserves over the axial compression of the ion beam pulse as long as the beam experiences linear forces. 


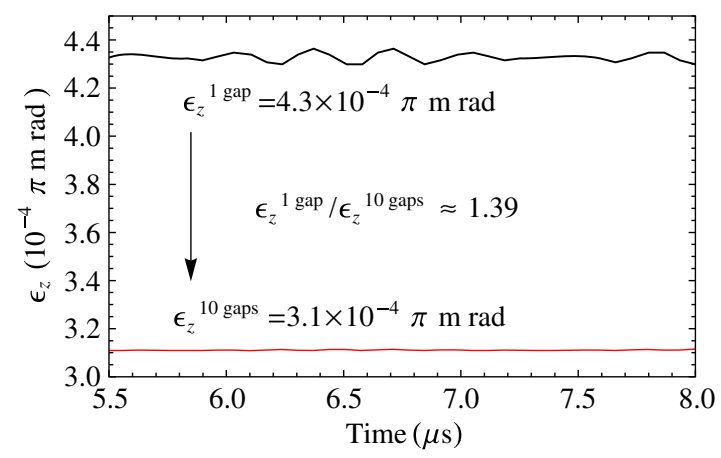

FIG. 3. The red curve shows the unnormalized longitudinal emittance $\epsilon_{z}\left(t \geq t_{a}=5.5 \mu \mathrm{s}\right)$ plot for the whole $\mathrm{Rb}$ beam accelerated by ten constant voltage gaps. The black curve represents the previous beam accelerated by one constant voltage gap in Fig. 2(a). $\epsilon_{z}^{10 \text { gaps }}$ is smaller than $\epsilon_{z}^{1 \text { gap }}$ by 1.39 times.

\section{B. Effect of multiple constant voltage gaps}

In contrast, the unnormalized longitudinal emittance decreases with the beam velocity. To illustrate this property, we passed a $\mathrm{Rb}$ beam through ten gaps, each has $0.2 \mathrm{MV}$ flattop voltage, to double the beam energy from 2 to $4 \mathrm{MeV}$, as shown by the red curve in Fig. 3. The black curve in Fig. 3 represents the previous beam discussed in Fig. 2(a). In a comparison between the beams passing through 10 and 1 acceleration gap, we found that the ratio $\epsilon_{z}^{1 \text { gap }} / \epsilon_{z}^{10 \text { gaps }}$ is about 1.39 , which is close to the factor $\sqrt{2 / 1.1} \approx 1.35$ according to Eq. (1). Since $\epsilon_{z}$ is proportional to $1 / v_{z}$, the factor of velocity is correlated to other factors such as the voltage fluctuation in the studies of longitudinal emittance growth during beam accelerations.

\section{Effect of time-varying voltage fluctuation gap}

We now proceed to study the time-varying voltage fluctuation gap assuming a simple sinusoidal voltage variation. The acceleration gap has constant voltage $\Phi_{0}=0.2 \mathrm{MV}$, amplitude of voltage variation $\delta \Phi$, and frequency $f_{n} \equiv$ $n / \tau$, where $\tau$ is the pulse length:

$$
\begin{gathered}
\Phi(t)=\Phi_{0}+\delta \Phi \sin \left(2 \pi f_{n} t\right), \\
v_{z}(z)=v_{z 0}+\delta v_{z} \sin \left(k_{n} z\right) .
\end{gathered}
$$

Here $v_{z 0}$ is the average beam velocity, beam length $L=$ $v_{z 0} \tau, k_{n}=2 n \pi / L, z=v_{z 0} t$, and $\delta v_{z}=v_{z 0} \delta \Phi / 2 \Phi_{0}$. We assume the line charge density distribution is uniform over the beam length $L$ such that

$$
\begin{gathered}
\left\langle\Delta z^{2}\right\rangle=L^{2} / 12, \quad\left\langle\Delta v_{z}^{2}\right\rangle=\delta v_{z}^{2} / 2, \quad\left\langle\Delta z \Delta v_{z}\right\rangle^{2}=\delta v_{z}^{2} / k_{n}^{2} \\
\epsilon_{z n, \delta}=\frac{4 L \delta v_{z}}{c} \sqrt{\frac{1}{24}-\frac{1}{4 n^{2} \pi^{2}}}=\frac{2 L \delta v_{z}}{c} \sqrt{\frac{1}{6}-\frac{1}{n^{2} \pi^{2}}} \\
\therefore \epsilon_{\mathrm{zn}, \delta}=L \delta_{\Phi} V_{z 0} G(n)
\end{gathered}
$$

where $G(n)=\frac{1}{c} \sqrt{\frac{1}{6}-\frac{1}{n^{2} \pi^{2}}}$ and $\delta_{\Phi} \equiv \frac{\delta \Phi}{\Phi}=2 \frac{\delta v_{z}}{v_{z 0}}$.

Equation (9) shows that the normalized longitudinal emittance grows explicitly with the fraction of voltage variation $\delta_{\Phi}$, hence the beam quality is worsened by the existence of voltage fluctuations.

Figure 4(a) represents the scenario of passing a $\mathrm{Rb}$ beam through a time-varying voltage fluctuation gap, located at a certain downstream position in the pipe. The voltage profile is designed according to Eq. (6), which has flattop voltage $\Phi_{0}=0.2 \mathrm{MV}$, frequency $f_{n=10} \sim 3.7 \mathrm{MHz}$, and the amplitude of voltage variation is $\delta \Phi$. In this figure, the black curve represents a $\mathrm{Rb}$ beam accelerated by a timevarying voltage fluctuation gap with $\delta \Phi=0.4 \mathrm{kV}$. We found that the normalized longitudinal emittance after the beam completely left the gap is well approximated by $\epsilon_{z n, \delta}=2.13 \times 10^{-5} \pi \mathrm{m} \mathrm{rad}$, which is about $73 \%$ of $\epsilon_{z n}$ obtained from the simulation. In contrast with the assumption of uniform line charge density distribution over the entire beam, the initial line charge density profile of the input $\mathrm{Rb}$ beam in our simulations has rounded ends with a flattop that extends $60 \%$ of the length of the beam, and with the flattop center coinciding with the beam center, which accounts for the slight difference between $\epsilon_{z n, \delta}$ and the simulation result. The red curve in the same figure
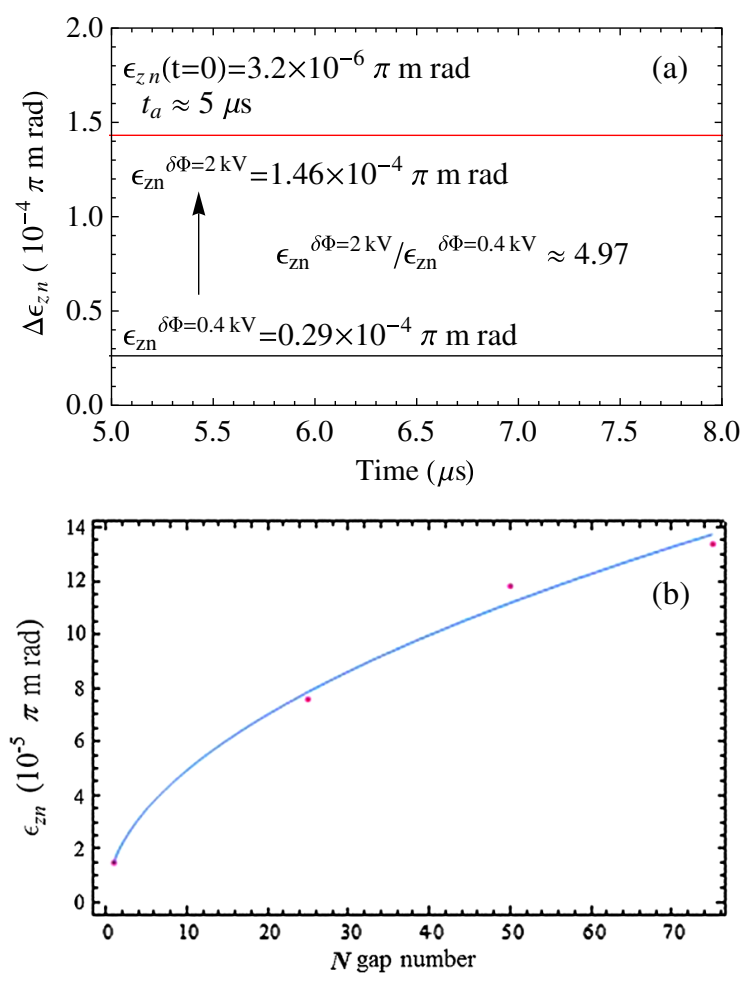

FIG. 4. (a) $\Delta \epsilon_{z n}(t)$ plot: the red curve represents the gap with $\delta \Phi=2 \mathrm{kV}$ and the black curve represents the gap with $\delta \Phi=$ $0.4 \mathrm{kV}$. (b) $\epsilon_{z n}(t)$ plot for Rb beams accelerated by a different number of time-varying voltage fluctuation gaps, shown by the red dots. The blue curve is the best-fit curve for these four data points. 
represents the case of $\delta \Phi=2 \mathrm{kV}, 5$ times larger than that in the case of $\delta \Phi=0.4 \mathrm{kV}$. Since longitudinal emittance is proportional to the amplitude of voltage variation $\delta \Phi$ according to Eq. (9), $\epsilon_{z n}$ for the beam accelerated by this gap should rise 5 times larger than that of the previous case. In Fig. 4(a), we found that the emittance growth of the red curve was about $\sim 5$ times larger than the black curve, which agrees with the relation $\epsilon_{z n, \delta} \sim \delta_{\Phi}$ in Eq. (9). The issue of time-varying fluctuations in acceleration voltages is common such as in the acceleration of ion beams as envisioned for heavy ion fusion, the longitudinal emittance growth due to gap voltage variation becomes unavoidable.

In general, the longitudinal emittance growth is huge as the beam continually accelerated by time-varying voltage fluctuation gaps. For example in Fig. 4(b), we consider the scenario of a superposition of time-varying sinusoidal voltage fluctuation gap with a random frequency distribution and random phases. Four red dots in this figure represent the $\mathrm{Rb}$ beam accelerated by $1,25,50$, and 75 time-varying voltage fluctuation gaps in four separate simulations, respectively. We found that $\epsilon_{z n}$ grows rapidly with $N$ the number of time-varying voltage fluctuation gaps, and agrees with the best-fit curve in relation $\epsilon_{z n} \sim \sqrt{N}$, the blue curve in Fig. 4(b). In this example, the voltage fluctuations are totally random so that the overall effective beam velocity fluctuation due to a single time-varying voltage fluctuation gap is either an increment $+\Delta v$ or a decrement $-\Delta v$. As for $N$ time-varying voltage fluctuation gaps, the pattern of emittance growth is similar to the random walk after $N$ steps so one expects $\epsilon_{z n}$ grows with the factor of $\sqrt{N}$.

\section{ONE-STEP CORRECTION}

The longitudinal emittance growth in the previous example of multigap acceleration with random voltage fluctuations is dominated by two factors: the voltage variation $\delta \Phi$ and the number of gaps $N$. Even if the magnitude of voltage variation $\delta \Phi$ of a single gap was minimized, the continual growth of longitudinal emittance with the factor of $\sqrt{N}$ will still lead to an unacceptably large value after successive ion beam accelerations. Techniques to reduce longitudinal emittance growth are thus desired. In "one-step correction" the velocity fluctuation is removed. It is based on the idea to measure and remove velocity fluctuations directly by imposing an opposite voltage to flatten the velocity fluctuation. The correction voltage profile can be technically realized by imposing a time-varying voltage modulation across an induction gap. For instance, the induction module in the NDCX beam line (Ref. [7]) consists of 14 ferromagnetic cores. Each core is driven independently by a thyratron-switched modulator, which is able to produce its own voltage waveform. Hence, the prescribed correction voltage profile at the induction gap can be obtained by using these 14 discrete cores. Figure 5 shows the setup for the one-step correction in WARP simulation. In this figure, a time-varying voltage fluctuation gap

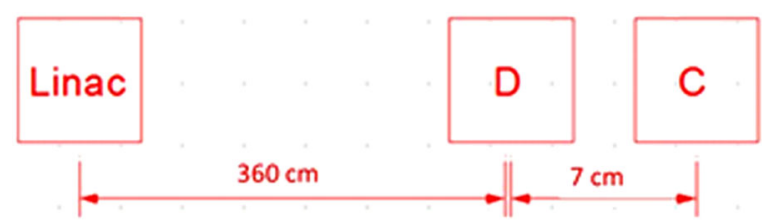

FIG. 5. Setup for the one-step correction. D is the position for velocity diagnostic and $\mathrm{C}$ is the position for the velocity correction.

called "Linac" is located at the pipe; a noninvasive beam energy diagnostic called D and a correction gap called C are put at a certain downstream distance next to each other. The voltage profile of Linac is designed in the same way in Sec. II C, but the sinusoidal voltage variation is now replaced by a superposition of sinusoidal fluctuations with frequency varying from 0 to $3.7 \mathrm{MHz}$ and random phases.

For the purpose of this paper, we assume that a precise diagnostic for the beam energy profile exists. [We showed in a separate paper (Ref. [8]) that a noninvasive energy diagnostic is in fact possible at any nonrelativistic energy.] The beam kinetic energy is measured at $\mathrm{D}$, and the corresponding beam velocity profile at $\mathrm{C}$ is calculated by the static time of flight using the velocity data obtained at $\mathrm{D}$. To remove all velocity fluctuations, an opposite velocity waveform is imposed at $\mathrm{C}$ to flatten the velocity fluctuation. In one-step correction, the density fluctuation remains uncorrected.

Figures 6(a) and 6(b) and the red curve in 6(c) represent the scenario of passing a $\mathrm{Rb}$ beam through the time-varying voltage fluctuation gap Linac, then the beam is corrected by the one-step correction. Figure 6(a) shows the disturbed beam velocity profile detected by the beam energy diagnostic located at D. Figure 6(b) shows the opposite velocity waveform applied at $\mathrm{C}$ to remove the previous velocity fluctuations detected at D. Under the one-step correction, the longitudinal emittance of the Rb beam shown by the red curve in Fig. 6(c) was reduced significantly. The performance of one-step correction is checked in Fig. 6(c). In this figure, the black curve represents the scenario of passing the $\mathrm{Rb}$ beam through the Linac but no correction is implemented. The longitudinal emittance of this uncorrected beam after completely leaving the gap at time $8 \mu \mathrm{s}$ is $\epsilon_{z n}{ }^{\text {uncorrected }}(t=8 \mu \mathrm{s})=12.79 \times 10^{-6} \pi \mathrm{m} \mathrm{rad}$. In comparison with the $\mathrm{Rb}$ beam accelerated by the Linac but corrected by the one-step correction shown by the red curve in Fig. 6(c), the longitudinal emittance for this corrected beam at the same moment is $\epsilon_{z n}{ }^{\text {corrected }}(t=8 \mu \mathrm{s})=$ $3.226 \times 10^{-6} \pi \mathrm{m} \mathrm{rad}$, a reduction of $75 \%$ was observed. Hence, the longitudinal emittance growth is correctable. High current beams are corrected in Sec. IV.

\section{A. Criteria for one-step correction}

The one-step correction generally works well for low current beams, since the space-charge wave effect is so 

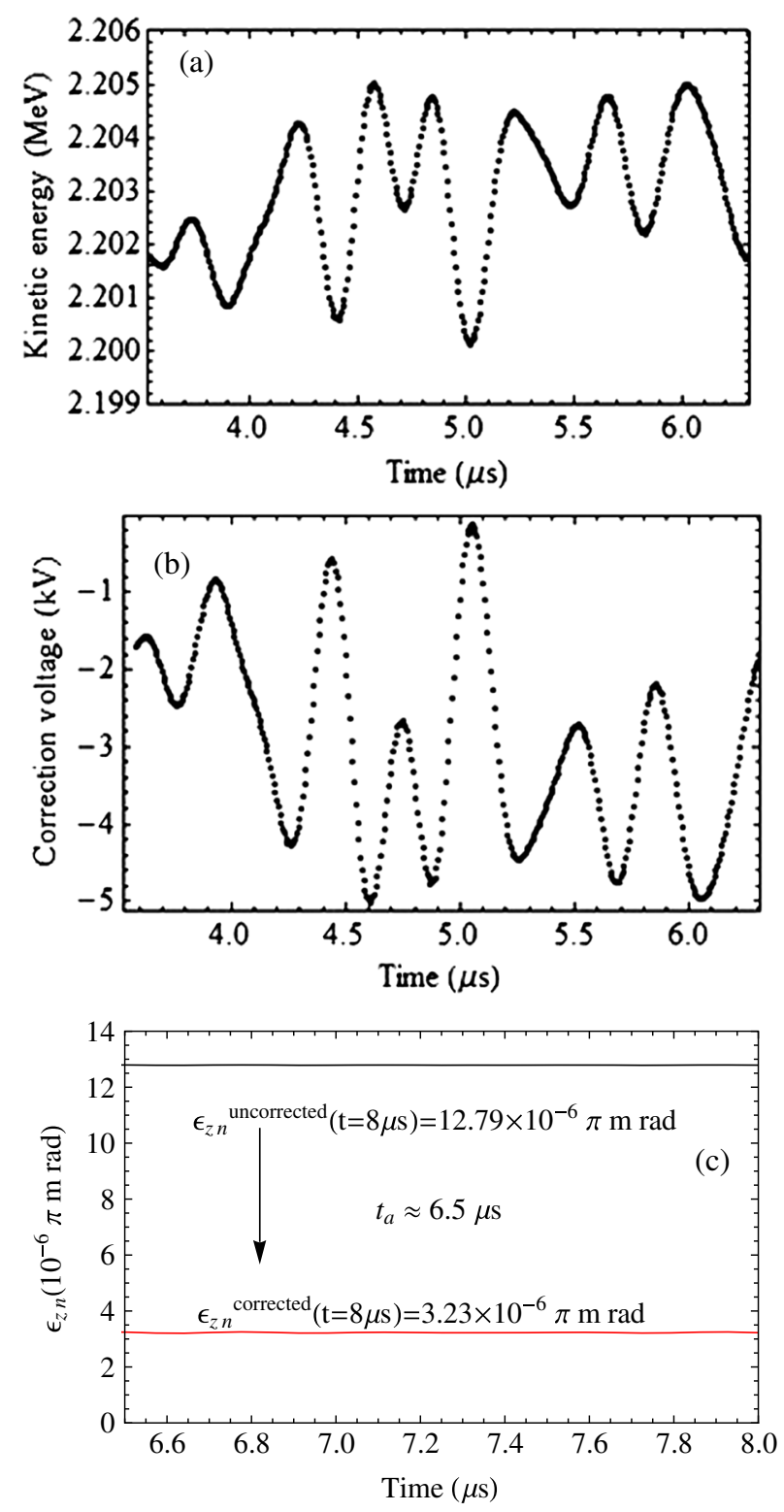

FIG. 6. (a) Beam kinetic energy profile detected at D. (b) Correction voltage profile applied at C. (c) $\epsilon_{z n}\left(t \geq t_{a}\right)$ plot for the $\mathrm{Rb}$ beam after the one-step correction.

weak that the assumption of static time of flight of velocity profiles holds. But the assumption is limited by the occurrence of particle overtaking. To minimize the cost to set up one-step corrections in multigap accelerations, any two pairs of one-step correction unit-a beam energy diagnostic $\mathrm{D}$ and a correction gap $\mathrm{C}$-must be put at the farthest separation right before the occurrence of particle overtaking.

To estimate the critical downstream distance before the particle overtaking, we assume $N$ particles of the initial beam are uniformly distributed over the beam length $L$, and a sinusoidal velocity fluctuation $v_{1}$ with wavelength $\lambda=2 L$ is imposed to the beam. The initial $v_{z}-z$ phase space configuration is shown by the blue dots in Fig. 7. The red dots in this figure show the state of particle overtaking.

At time $t=0$ in the beam frame, each particle labeled by index $n$ is located $z_{n}=n L / N$ with sinusoidal velocity fluctuation

$$
v_{1}\left(z_{n}\right)=\delta_{v} \sin \left(k z_{n}\right),
$$

where $\delta_{v}$ is the amplitude of velocity variation and $k=$ $2 \pi / \lambda$. After time $\Delta t=\Delta z / v_{z 0}$, where $\Delta z$ is the longitudinal displacement of the beam center measured in the lab frame, each particle moves to

$$
\tilde{z}_{n}=z_{n}+v_{1}\left(z_{n}\right) \Delta t
$$

The particle overtaking appears when

$$
(d \tilde{z} / d n)_{n=N}=0 .
$$

In terms of $\lambda_{m}=\frac{\lambda_{0}}{m}=\frac{\tau_{0} v_{z 0}}{m}=\frac{v_{z 0}}{f_{m}}$, define $P \equiv \frac{\delta_{v}}{2 v_{z 0}}$ :

$$
\therefore \mathrm{z}(\text { critical distance }) \approx \frac{\boldsymbol{v}_{z 0}}{\pi f_{m} P} \text {. }
$$

For example, in Figs. 8(a) and 8(b) a Rb beam is accelerated by successive gaps with this prescribed sinusoidal velocity variation at an interval of $0.72 \mathrm{~m}$. Each gap has $0.2 \mathrm{MV}$ flattop voltage, and the voltage fluctuation is in a superposition of different sinusoidal waves with amplitude $\delta \Phi=2 \mathrm{kV}$, frequency spectrum varying from 0 to $f_{10} \approx$ 3.7 $\mathrm{MHz}$ and random phases. From the $v_{z}-z$ phase space plots in Figs. 8(a) and 8(b), we found that the particle overtaking appears after 70 gaps, which means the longest separation to install the next one-step correction unit in this example is about $\Delta z_{\text {simulation }}=70 \times 0.72 \approx 50.4 \mathrm{~m}$. To apply Eq. (13) for the critical distance, the highest frequency component is used because particle overtaking appears earlier for short wavelength modes. In this example, we have $f_{m=10}=3.7 \times 10^{6} \mathrm{~Hz}$ and $P=$ $2.5 \times 10^{-3}$ so the estimated critical distance is $\Delta z_{\text {critical }} \approx$ $73 \mathrm{~m}>\Delta z_{\text {simulation }} . \Delta z_{\text {critical }}$ overestimates the critical distance, because the beam in simulation was continually accelerated by gaps so that the effective amplitude of

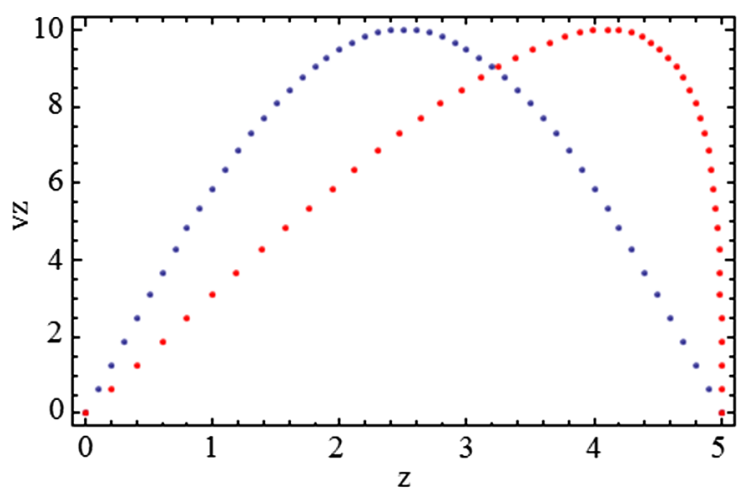

FIG. 7. An example of particle overtaking in $v_{z}-z$ phase space in the beam frame. 

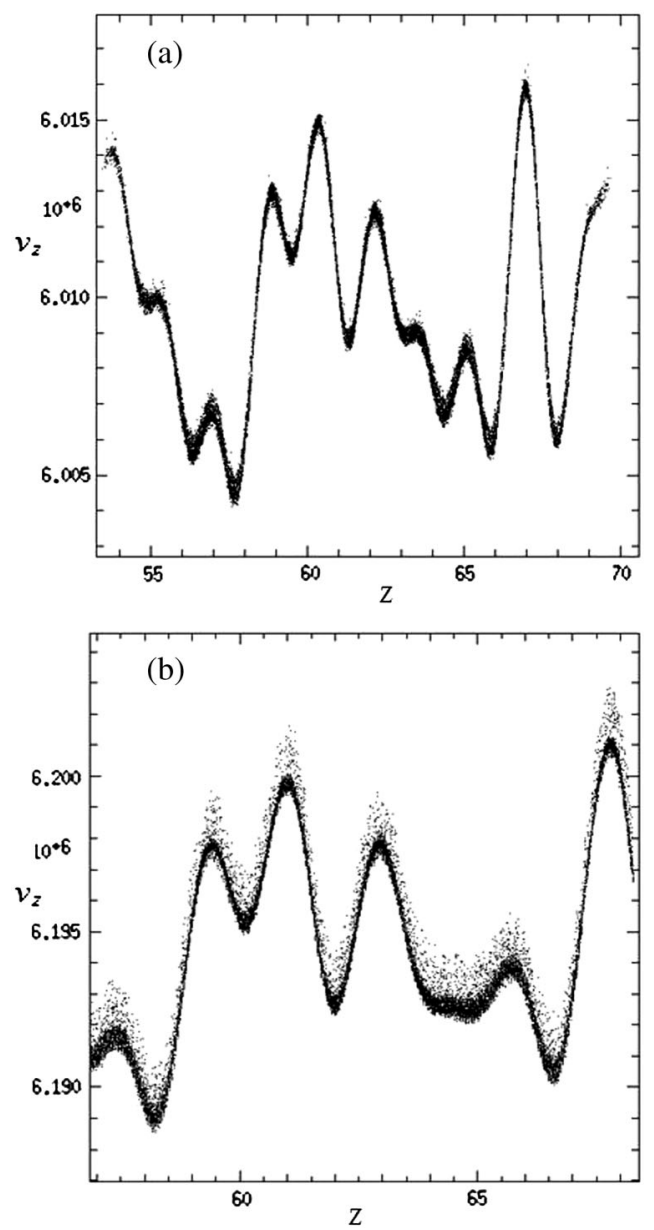

FIG. 8. $v_{z}-z$ phase space after (a) 70 gaps; (b) 75 gaps.

velocity variation $\delta_{v}$ is increasing with the number of gaps, resulting in shorter critical distance in simulation.

\section{B. Space-charge dominated beam at high current}

The assumption of static time of flight of the velocity profile over $7 \mathrm{~cm}$ in the previous example of one-step correction is inaccurate if velocity fluctuations evolve with time. Space-charge waves are significant in high current beams and dominate the evolution of velocity and density fluctuations. In Fig. 9(a), the growth of $\epsilon_{z n}$ after the one-step correction for a $0.02 \mathrm{~mA}$ low current $\mathrm{Rb}$ beam is compared with a 0.02 A high current $\mathrm{Rb}$ beam. Since the density fluctuation remains uncorrected, the evolution of density waves in the high current beam continually contributes to the longitudinal emittance growth. Consequently, the longitudinal emittance for the $0.02 \mathrm{~A}$ high current $\mathrm{Rb}$ beam represented by the red curve in Fig. 9(a) grows immediately after the one-step correction. In contrast, the longitudinal emittance for the $0.02 \mathrm{~mA}$ low current $\mathrm{Rb}$ beam represented by the black curve in the same figure remains nearly constant after the correction.

In addition, the continual growth of the big velocity difference between the head and the tail of a space-charge
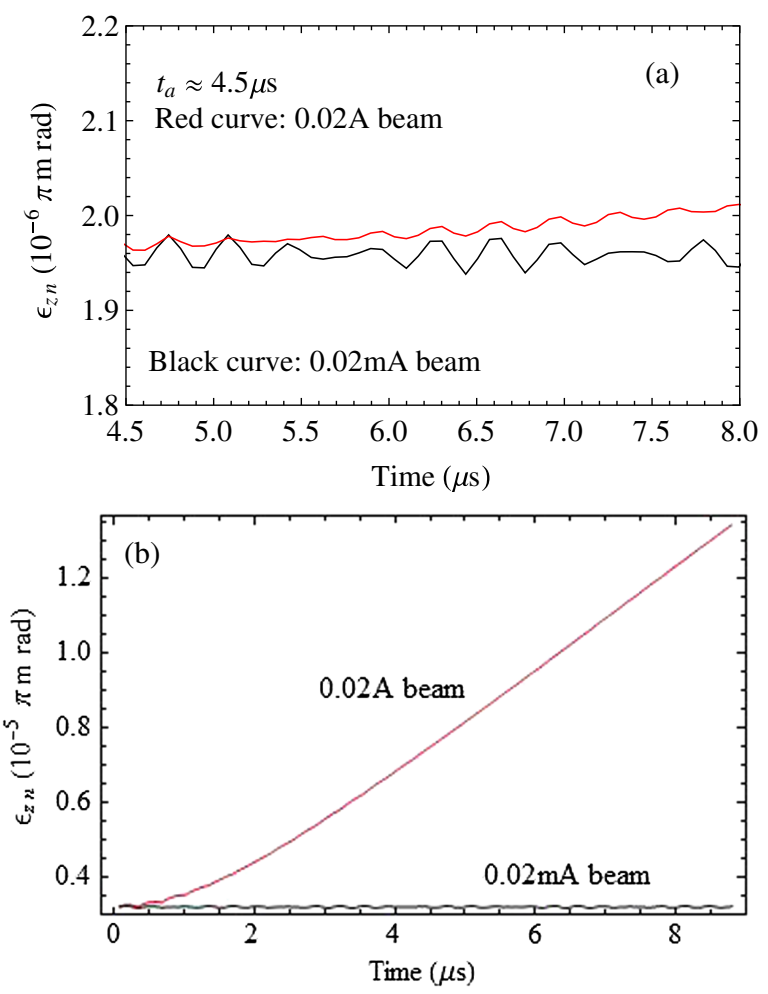

FIG. 9. (a) $\epsilon_{z n}\left(t \geq t_{a}\right)$ plot for a $0.02 \mathrm{~mA}$ low current $\mathrm{Rb}$ beam (black curve) after the one-step correction is compared with a $0.02 \mathrm{~A}$ high current $\mathrm{Rb}$ beam (red curve). $t_{a}$ is the moment when the beam completely left the last gap. (b) Compares the longitudinal emittance growth due to the head-to-tail nonlinearity of a $0.02 \mathrm{~mA}$ low current $\mathrm{Rb}$ beam (black curve) with a $0.02 \mathrm{~A}$ high current $\mathrm{Rb}$ beam (red curve).

dominated beam contributes further emittance growth. Figure 9(b) compares the longitudinal emittance growth due to the head-to-tail nonlinearity of a $0.02 \mathrm{~mA}$ low current, coasting inside the pipe without any gap, with a 0.02 A high current $\mathrm{Rb}$ beam. However, the effect of headto-tail nonlinearity (Ref. [9]) is not treated in this paper, and it can be avoided by adding "ear fields" to keep the head and tail at constant energy over time. In the rest of the paper, all simulations for high current beams were imposed with ear fields.

\section{TWO-STEP CORRECTION}

\section{A. General evolution of space-charge waves}

To understand the dynamics of velocity and density waves, the general evolution of space-charge waves in time and space is derived here based on the weak perturbation of the 1D cold fluid model (Refs. [10-12]). Then the wave solutions are used to compute required correction voltages to remove both velocity and density fluctuations.

Longitudinal continuity and momentum equations are

$$
\frac{\partial \lambda}{\partial t}+\frac{\partial\left(\lambda v_{z}\right)}{\partial z}=0
$$




$$
\frac{\partial v_{z}}{\partial t}+v_{z} \frac{\partial v_{z}}{\partial z}=\frac{q}{m} E_{z}-\frac{1}{\rho} \frac{\partial P}{\partial z} .
$$

Here $\lambda$ is the line charge density, $q$ the ion charge, $m$ the ion mass, and $E_{z}$ the longitudinal self-electric field,

$$
E_{z} \approx-g \frac{\partial \lambda}{\partial z}
$$

where $g=\frac{1}{2 \pi \epsilon_{0}} \ln (b / a)$.

Here $b / a$ is the ratio of the pipe radius to the mean beam radius and $g$ is the $g$ factor. Substitute the pressure term $P=\rho k T / m$ into the momentum equation,

$$
\frac{\partial v_{z}}{\partial t}+v_{z} \frac{\partial v_{z}}{\partial z}=-\left(\frac{q g \lambda}{m}\right) \frac{1}{\lambda} \frac{\partial \lambda}{\partial z}-\left(\frac{k T}{m}\right) \frac{1}{\rho} \frac{\partial \rho}{\partial z} .
$$

In fact, the pressure term on the right-hand side of the momentum equation can be neglected provided that $k T / m \ll q g \lambda / m$, which in turn requires high generalized perveance ion beams injected from the source at low temperature. Since the source temperature is small in our simulations, it is valid to neglect the pressure term. By substituting $v_{z}=v_{z 0}+v_{1}, \lambda=\lambda_{0}+\lambda_{1}$, and taking the first order approximation, a wave equation for the perturbed line charge density $\lambda_{1}$ is obtained, which propagates with the space-charge wave speed $c_{s}$. The solution of velocity wave $v_{1}$ can be obtained by considering Eq. (14) after solving for $\lambda_{1}$ :

$$
\frac{\partial^{2} \lambda_{1}}{\partial t^{\prime 2}}-c_{s}^{2} \frac{\partial^{2} \lambda_{1}}{\partial z^{\prime 2}}=0
$$

where $c_{s}=\sqrt{\frac{q g \lambda_{0}}{m}}$.

The subscript 0 represents the equilibrium value while the subscript 1 represents the perturbed part of that quantity. $t^{\prime}$ and $z^{\prime}$ are measured in the beam frame but laboratory frame variables $t$ and $z$ are used in the rest of the paper. The general wave solution is a pair of a forward-moving wave $\xi_{+}$and a backward-moving wave $\xi_{-}$and they are solved completely by acquiring well-posed boundary conditions. General wave solutions are

$$
\begin{aligned}
& \lambda_{1}(t, z)=\lambda_{0}\left[\xi_{+}\left(t-\frac{z}{v_{z 0}+c_{s}}\right)+\xi_{-}\left(t-\frac{z}{v_{z 0}-c_{s}}\right)\right] \\
& v_{1}(t, z)=c_{s}\left[\xi_{+}\left(t-\frac{z}{v_{z 0}+c_{s}}\right)-\xi_{-}\left(t-\frac{z}{v_{z 0}-c_{s}}\right)\right] .
\end{aligned}
$$

Define gap voltage $\Phi \equiv \Phi_{0}+\Phi_{1}$ and $f_{i} \equiv\left(\frac{\Phi_{1}}{\Phi_{0}}\right)_{a t z_{i}}$.

Boundary conditions at $z=0$,

$$
v_{1}(t, z=0)=v_{1}(t), \quad \lambda_{1}(t, z=0)=\lambda(t) .
$$

Solving $\xi_{+}$and $\xi_{-}$gives

$$
\xi_{+}(t)=\frac{1}{2}\left[\frac{\lambda_{1}(t)}{\lambda_{0}}+\frac{v_{1}(t)}{c_{s}}\right], \quad \xi_{-}(t)=\frac{1}{2}\left[\frac{\lambda_{1}(t)}{\lambda_{0}}-\frac{v_{1}(t)}{c_{s}}\right]
$$

Define phase factor $\varphi_{ \pm}(z) \equiv z /\left(v_{z 0} \pm c_{s}\right)$, therefore the general wave solutions are

$$
\begin{aligned}
v_{1}(t, z)= & \frac{1}{2}\left[v_{1}\left(t-\varphi_{+}\right)+v_{1}\left(t-\varphi_{-}\right)\right] \\
& +\frac{c_{s}}{2 \lambda_{0}}\left[\lambda_{1}\left(t-\varphi_{+}\right)-\lambda_{1}\left(t-\varphi_{-}\right)\right], \\
\lambda_{1}(t, z)= & \frac{\lambda_{0}}{2 c_{s}}\left[v_{1}\left(t-\varphi_{+}\right)-v_{1}\left(t-\varphi_{-}\right)\right] \\
& +\frac{1}{2}\left[\lambda_{1}\left(t-\varphi_{+}\right)+\lambda_{1}\left(t-\varphi_{-}\right)\right] .
\end{aligned}
$$

\section{B. Principle of two-step correction}

Knowing the complete solutions of $\lambda_{1}$ and $v_{1}$ allows us to predict their waveform at any time and position, which in turn enables us to compute required correction voltages to generate opposite waveforms to remove density and velocity fluctuations. This technique is called two-step correction, in which the first correction voltage is used to remove density fluctuations and then the second correction voltage is used to remove velocity fluctuations.

Figure 10 shows the scenario for the two-step correction. First an unperturbed $\mathrm{Rb}$ beam that has no disturbance in both density and velocity profiles is loaded inside the pipe. Then it is accelerated by a time-varying voltage fluctuation gap $f_{0}(t)$ to excite space-charge waves. The first correction is implemented by applying a prescribed voltage $f_{1}(t)$ at $z_{1}$ to generate another density wave that exactly cancels the one generated by $f_{0}(t)$ at $z_{2}$. So all density fluctuations are removed right at $z_{2}$. At the same instant, the second correction is implemented by applying a prescribed voltage $f_{2}(t)$ at $z_{2}$ to generate another velocity wave that exactly cancels the one generated by $f_{0}(t)$ and $f_{1}(t)$ at $z_{2}$. Similarly all velocity fluctuations are removed right at $z_{2}$. Achieving zero $\lambda_{1}$ and $v_{1}$ simultaneously at one space-time point is crucial, because it ensures a boundary condition that has no space-charge wave excitation so that $\lambda_{1}$ and $v_{1}$ vanish after $z_{2}$ for all time. In our simulation studies, the distortion of wave solutions caused by waves reflected from the beam head and tail is avoided by placing the density and velocity correction voltages at a short distance from the first gap $f_{0}$ such that reflected waves do not have sufficient time to propagate into the beam body and the solutions of correction voltage profiles remain valid.

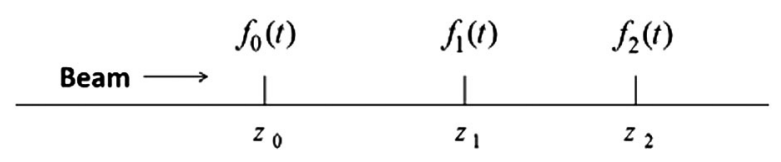

FIG. 10. Setup for the two-step correction. 


\section{Solving $f_{1}(t)$ for density fluctuation correction}

At $z_{0}$ the boundary conditions are

$\lambda_{1}(t)=0, \quad v_{1}(t)=\frac{v_{z 0}}{2}\left(\frac{\Phi_{1}(t)}{\Phi_{0}}\right)_{a t z_{0}}=\frac{v_{z 0}}{2} f_{0}(t)$.

When $f_{1}(t)$ is absent, the density wave at $z_{2}$ is

$\lambda_{1}\left(t, z_{2}\right)=\frac{\lambda_{0} v_{z 0}}{4 c_{s}}\left[f_{0}\left(t-\frac{z_{2}}{v_{z 0}+c_{s}}\right)-f_{0}\left(t-\frac{z_{2}}{v_{z 0}-c_{s}}\right)\right]$.

When $f_{1}$ is present, the density wave at $z_{2}$ is

$$
\begin{aligned}
\lambda_{1}\left(t, z_{2}\right)= & \frac{\lambda_{0} \boldsymbol{v}_{z 0}}{4 c_{s}}\left[f_{0}\left(t-\frac{z_{2}}{\boldsymbol{v}_{z 0}+c_{s}}\right)-f_{0}\left(t-\frac{z_{2}}{\boldsymbol{v}_{z 0}-c_{s}}\right)\right. \\
& \left.+f_{1}\left(t-\frac{z_{2}-z_{1}}{\boldsymbol{v}_{z 0}+c_{s}}\right)-f_{1}\left(t-\frac{z_{2}-z_{1}}{\boldsymbol{v}_{z 0}-c_{s}}\right)\right] .
\end{aligned}
$$

Define $F_{0}(t) \equiv f_{0}\left(t-\frac{z_{2}}{v_{z 0}+c_{s}}\right)-f_{0}\left(t-\frac{z_{2}}{v_{z 0}-c_{s}}\right)$, and $F_{1}(t) \equiv$ $f_{1}\left(t-\frac{z_{2}-z_{1}}{v_{z 0}+c_{s}}\right)-f_{1}\left(t-\frac{z_{2}-z_{1}}{v_{z 0}-c_{s}}\right)$.

Notice that the application of the voltage $f_{1}(t)$ excites another density wave $F_{1}(t)$, but this voltage $f_{1}(t)$ is constrained to give a zero density fluctuation at $z_{2}$,

$$
\lambda_{1}\left(t, z_{2}\right)=0 \quad \text { or } \quad F_{0}(t)+F_{1}(t)=0 .
$$

The above condition constrains the allowed waveform of $f_{1}(t)$ at $z_{1}$. To solve for $f_{1}(t)$, we expand $f_{1}(t)$ into Fourier series

$$
f_{1}(t)=\sum_{n=-\infty}^{+\infty} c_{n} e^{i \omega_{n} t}
$$

$\omega_{n}=2 n \pi / \tau$ and $\tau$ is the pulse length. In fact only Fourier components $c_{n}$ with large contribution to fluctuations are important so that the summation actually involves a finite range of $n$. On solving the lower of Eq. (24), $c_{n}$ is found:

$$
c_{n}=\frac{\frac{1}{T} \int_{t_{1}}^{t_{2}}-F_{0}(t) e^{-i \omega_{n} t} d t}{e^{-i \omega_{n}\left(\frac{z_{2}-z_{1}}{v_{z 0}+c_{s}}\right)}-e^{-i \omega_{n}\left(\frac{z_{2}-z_{1}}{v_{z 0} c_{s}}\right)}} .
$$

Here time interval $t_{1}-t_{2}$ is chosen, which means the duration as the beam passing through $z_{2}$.

\section{Solving $f_{\mathbf{2}}(t)$ for velocity fluctuation correction}

Since $f_{1}(t)$ is now known, the overall velocity waves generated by $f_{0}(t)$ and $f_{1}(t)$ are given by

$$
\begin{aligned}
\boldsymbol{v}_{1}\left(t, z_{2}\right)= & \frac{\boldsymbol{v}_{z 0}}{4}\left[f_{0}\left(t-\frac{z_{2}}{\boldsymbol{v}_{z 0}+c_{s}}\right)+f_{0}\left(t-\frac{z_{2}}{\boldsymbol{v}_{z 0}-c_{s}}\right)\right. \\
& \left.+f_{1}\left(t-\frac{z_{2}-z_{1}}{\boldsymbol{v}_{z 0}+c_{s}}\right)+f_{1}\left(t-\frac{z_{2}-z_{1}}{\boldsymbol{v}_{z 0}-c_{s}}\right)\right] .
\end{aligned}
$$

Finally, this velocity wave $v_{1}\left(t, z_{2}\right)$ is removed by imposing voltage $f_{2}$ that exactly cancels $v_{1}\left(t, z_{2}\right)$.

$$
f_{2}(t)=-\frac{v_{1}\left(t, z_{2}\right)}{v_{z 0}} \times 2 .
$$

\section{E. Simulation of two-step correction}

We now proceed to study the two-step correction technique by WARP simulations using a $0.02 \mathrm{~A}$ high current $\mathrm{Rb}$ beam. The time-varying voltage fluctuation gap $f_{0}(t)$ is designed as a superposition of different sinusoidal waves according to Eq. (29) and the flattop voltage is set to be zero $\Phi_{0}=0$ for simplicity.

$f_{0}(t)=N \sum_{n=1}^{10} \sin \left(\frac{2 n \pi}{\tau} t+\alpha_{n}\right)+\cos \left(\frac{2 n \pi}{\tau} t+\beta_{n}\right)$.

Here $N$ is a constant that makes the root mean square of $f_{0}(t)$ about $0.1 \%$ of the beam energy. $\alpha_{n}$ and $\beta_{n}$ are random phases assigned for each sinusoidal wave with frequency $\omega_{n}=2 n \pi / \tau$. We further assume the waveform of $f_{0}(t)$ is known and detectable. Since voltage $f_{1}(t)$ is used to achieve zero density fluctuation at $z_{2}, f_{1}(t)$ is called density error correction voltage (DEC). Similarly, voltage $f_{2}(t)$ is used to achieve zero velocity fluctuation at $z_{2} ; f_{2}(t)$ is called velocity error correction voltage (VEC).

\section{Performance of DEC at $z_{2}$}

Figure 11(a) shows the effect of DEC on the line charge density profile $\lambda(t)$ at $z_{2}$. The black curve represents an uncorrected $\mathrm{Rb}$ beam, in which an obvious density fluctuation was observed. The red curve in the same figure represents a $\mathrm{Rb}$ beam which was corrected by DEC voltage at $z_{1}$. A flattop was observed on the red curve which suggests that the density fluctuations at $z_{2}$ are effectively removed by DEC.

\section{Performance of VEC at $z_{2}+0.72 m$}

Figure 11(b) shows the effect of VEC on the velocity profile $v_{z}(t)$ at $z_{2}+0.72 \mathrm{~m}$. The black curve represents a $\mathrm{Rb}$ beam which was only corrected by DEC at $z_{1}$ while the red curve represents another $\mathrm{Rb}$ beam but it was corrected by a complete two-step correction: DEC at $z_{1}$ and VEC at $z_{2}$. The flattop observed on the red curve suggests that the velocity fluctuations at $z_{2}$ are effectively removed by VEC.

\section{Reduction of $\epsilon_{z n}$ after correction}

In Figs. 11(a) and 11(b), we found that both density and velocity fluctuations were effectively removed by the twostep correction, so the longitudinal emittance growth is suppressed. Figure 12(a) shows the growth of $\epsilon_{z n}$ for two $\mathrm{Rb}$ beams in different scenarios. The red curve represents a $0.02 \mathrm{~A} \mathrm{Rb}$ beam after the two-step correction while the black curve represents an uncorrected $0.02 \mathrm{~A} \mathrm{Rb}$ beam after accelerated by the time-varying voltage fluctuation gap $f_{0}(t)$. In this figure we found that the uncorrected beam has a large value of longitudinal emittance 

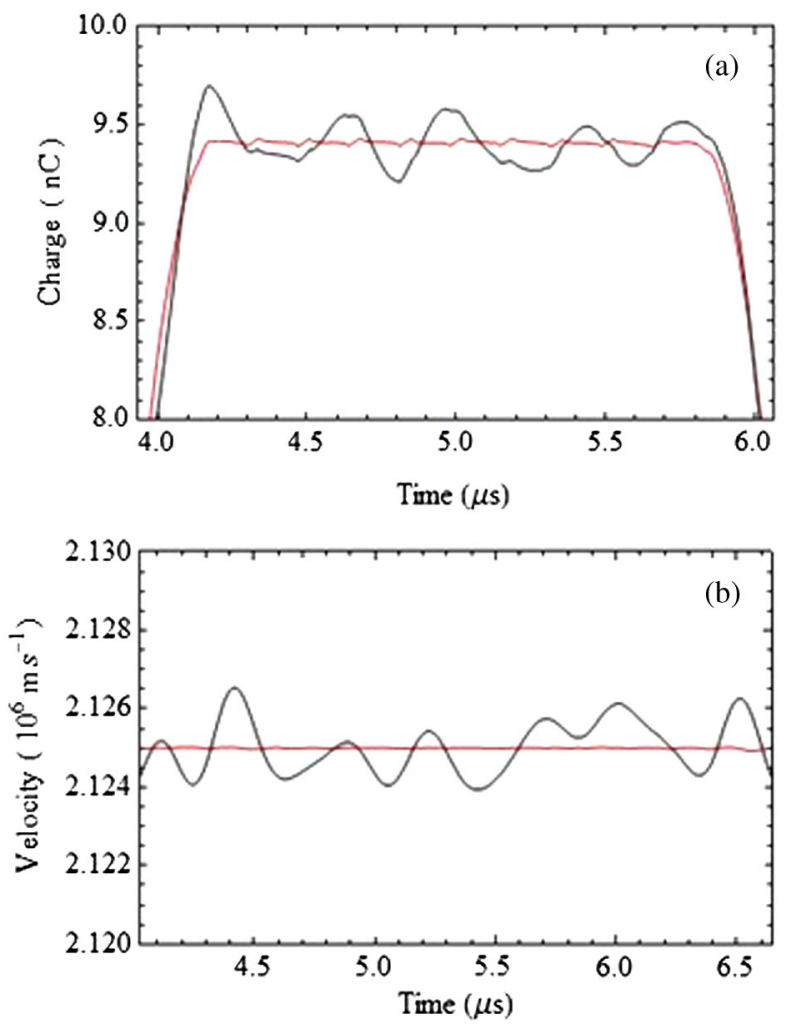

FIG. 11. (a) The line-charge density profile to show the performance of DEC at $z_{2}$. (b) The beam velocity profile to show the performance of VEC at $z_{2}+0.72 m$.

$\epsilon_{z n}^{\text {uncorrected }}\left(t_{a}=5.8 \mu \mathrm{s}\right) \approx 5.6 \times 10^{-6} \pi \mathrm{m} \mathrm{rad}$, but compared with the corrected beam after two-step correction, the longitudinal emittance is $\epsilon_{z n}{ }^{\text {corrected }}\left(t_{a}=5.8 \mu \mathrm{s}\right) \approx$ $2.0 \times 10^{-6} \pi \mathrm{m} \mathrm{rad}$, hence $\epsilon_{z n}$ was reduced by $64 \%$.

Figure 12(b) compares the performance of the two-step correction with the one-step correction for $0.02 \mathrm{~A}$ high current $\mathrm{Rb}$ beams. Since the density fluctuation is not corrected in the one-step correction represented by the blue curve in this figure, the growing density wave leads to an immediate slightly growth of $\epsilon_{z n}$ from $\epsilon_{z n}{ }^{1 \operatorname{step}}(5.8 \mu \mathrm{s}) \approx 1.98 \times 10^{-6} \pi \mathrm{mrad}$ to $\epsilon_{z n}{ }^{1 \operatorname{step}}(8 \mu \mathrm{s}) \approx$ $2.01 \times 10^{-6} \pi \mathrm{mrad}$. The density waves then induce velocity waves. After a sufficient long time, a large longitudinal emittance growth appears again. In contrast, the longitudinal emittance growth for the beam corrected by the two-step correction represented by the red curve in this figure remained nearly constant. Clearly, two-step correction is preferred because, ideally, it could suppress the growth of further space-charge waves after the correction.

Induction cells typically impose overall voltage increments of 100 's of $\mathrm{kV}$, so that voltage errors may be in the 0.1 to $1 \mathrm{kV}$ range. This contrasts to typical kinetic temperatures of $0.1 \mathrm{eV}$ at the source, so it is quite typical that voltage errors will induce velocity errors that are much larger than the thermal velocities in the beam. Space-
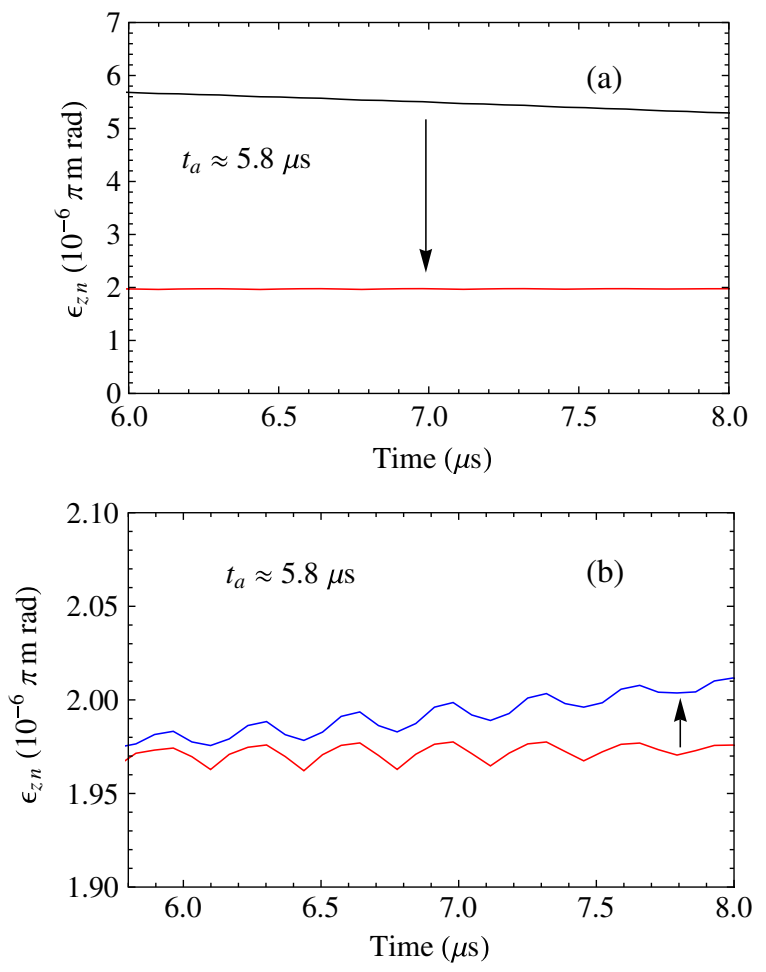

FIG. 12. (a) $\epsilon_{z n}\left(t \geq t_{a}\right)$ plot to compare the performance of two-step correction (red curve) with a uncorrected $\mathrm{Rb}$ beam (black curve). (b) $\epsilon_{z n}\left(t \geq t_{a}\right)$ plot to compare the performance of one-step correction (blue curve) with the two-step correction (red curve) for $0.02 \mathrm{~A}$ high current $\mathrm{Rb}$ beams. $t_{a}$ is the moment when the beam completely left the last gap.

charge waves are generated by these voltage errors and the width of the velocity distribution will be dominated by the space-charge waves rather than by the intrinsic temperature of the source. In the absence of correction, the waves will eventually phase mix and in practice the longitudinal emittance will be determined by the waves induced by voltage errors. The analysis assumes a longitudinal "cold" fluid approximation, which amounts to neglecting the pressure term in the fluid momentum equation. This is equivalent to assuming $k T / m \ll$ $q g \lambda / m$, where $k T$ is the ion temperature in the beam frame. We may express this as $\Delta E / E \ll \sqrt{8 \pi \epsilon_{0} g Q}$, where $Q$ is the generalized perveance and $E$ is the beam energy. For $Q$ in the range of $10^{-5}-10^{-3}$ (typical of the space-charge dominated beams needed for the application in heavy ion fusion) this corresponds to $\Delta E / E<10^{-2}-10^{-1}$ respectively, an energy spread far larger than could be tolerated for the application. We also assumed the $g$-factor model for the electric field which is a long wavelength approximation requiring a wavelength perturbation $L$ to be much greater than the beam radius $a$. This corresponds to breakdown of the model for frequencies greater than $3 \times 10^{10} \mathrm{~Hz}$ $(1 \mathrm{~cm} / a) \beta$, which is usually above frequencies of interest. Finally, we have assumed nonrelativistic beams 
$(\beta \ll 1)$ in our formulation. We expect the methods shown here can be generalized so that all three of these approximations could be improved upon to extend the validity of the work.

A two-step correction technique is developed based on the 1D cold fluid model. Since the use of 1D equations are sufficient to remove much of the emittance growth caused by the voltage fluctuations, a 3D code is used to calculate the residual level of fluctuations. In addition, the goal of the correction techniques is to reduce the growth of longitudinal emittance for real ion beams caused by voltage fluctuations. Therefore, a 3D code is used so that it can validate the formulation of the two-step correction by including all essential physics of a real ion beam.

\section{CONCLUSION}

We showed that whenever energy and current profiles are measured after a series of gaps by noninvasive means, space-charge wave dynamics can be solved completely and fluctuations in velocity and density profiles can be removed by imposing appropriate correction voltages. Therefore, both techniques of one-step and two-step corrections succeed to reduce $\epsilon_{z n}$ significantly. Even in space-charge dominated beams, the two-step correction was shown workable to achieve a significant reduction of $\epsilon_{z n}$, implying that the fundamental limit on pulse length at the source can be relaxed in the near term as well as driver-scale experiments of heavy ion fusion. In addition, these correction techniques can be extended for high energy density physics and other intense beam applications whenever time-varying voltage fluctuations appear.
[1] P. A. Seidl, E. P. Lee, R. O. Bangerter, and A. Faltens, Lawrence Berkeley National Laboratory Report No. LBNL-3101E, HIFAN 1768, 2010.

[2] S. S. Yu, W. R. Meier, R. P. Abbott, J. J. Barnard, T. Brown, D. A. Callahan, P. Heitzenroeder, J. F. Latkowski, B. G. Logan, S. J. Pemberton, P. F. Peterson, D. V. Rose, G. L. Sabbi, W. M. Sharp, and D. R. Welch, Fusion Sci. Technol. 44, 266 (2003) [http://www.ans.org/pubs/journals/fst/a_345].

[3] M. Reiser, Theory and Design of Charged Particle Beams (Wiley-VCH Weinheim, Germany, 2008), 2nd ed., Sec. 6.3.2.

[4] E.P. Lee and R. K. Cooper, Part. Accel. 7, 83 (1976) [http://www.osti.gov/energycitations/product.biblio.jsp? osti_id=7142237].

[5] O. A. Anderson, Phys. Rev. ST Accel. Beams 10, 034202 (2007).

[6] S. Humphries, Principles of Charged Particle Acceleration (John Wiley and Sons, New York, 1999), Chap. 7-8; Charged Particle Beams (John Wiley and Sons, New York, 1990), Chap. 3-4.

[7] P. K. Roy et al., in Proceedings of the 21st Particle Accelerator Conference, Knoxville, 2005 (IEEE, Piscataway, NJ, 2005), pp. 3856-3858.

[8] K. M. Woo and S. S. Yu, Nucl. Instrum. Methods Phys. Res., Sect. A 690, 34 (2012).

[9] Y. Y. Lau, M.Phil. thesis, The Chinese University of Hong Kong, 2011.

[10] J. J. Barnard, Lawrence Berkeley National Laboratory Report No. LBNL-56504, HIF Note No. 96-12, 1996.

[11] J. G. Wang, D. X. Wang, D. Kehne, and M. Reiser, in Proceedings of the Particle Accelerator Conference, Washington, DC, 1993 (IEEE, New York, 1993), Vol. 5, pp. 3282-3284.

[12] K. Tian, R. A. Kishek, I. Haber, M. Reiser, and P. G. O'Shea, Phys. Rev. ST Accel. Beams 13, 034201 (2010). 